\title{
DESARROLLO DE UNA APLICACIÓN WEB PARA EL MONTAJE DE UNA MESA QUIRÚRGICA EN EL ÁREA DE TRAUMATOLOGÍA
}

\author{
DEVELOPMENT OF A WEB APPLICATION FOR A SURGICAL INSTRUMENTS KIT \\ INSIDE THE TRAUMATOLOGY AREA
}

\author{
Diego Tangarife Chalarca
}

\section{Resumen}

El creciente desarrollo y evolución de las tecnologías, unido a los grandes cambios sociales han propiciado el replanteamiento en los sistemas tradicionales de enseñanza, donde el profesor asumía el rol principal. En la actualidad, existen diversas áreas donde la tecnología ha revolucionado las formas de impartir conocimiento, jugando un papel transformador e innovador en los procesos educativos. En esta investigación se describe el diseño de una aplicación web como estrategia para el aprendizaje de instrumental quirúrgico en el área de traumatología. La arquitectura de desarrollo solo incluyó componentes de código abierto, por lo que esta versión es totalmente software libre y se puede liberar con cualquier tipo de licencia de código abierto. Igualmente, se presenta el estado del arte de las herramientas existentes para la enseñanza y entrenamiento del personal médico en el área quirúrgica, especialmente en el campo de la cirugía traumatológica. El trabajo concluye con algunas recomendaciones que se deben tener en cuenta a la hora de desarrollar este tipo de aplicaciones.

Palabras clave: Aplicación web, juegos serios, instrumental quirúrgico, entrenamiento quirúrgico.

\begin{abstract} Aceptado: 20 de diciembre de 2013

The growing development and evolution of technologies along with major social changes have fostered a reframing in traditional teaching systems where, until now, the professor assumed the leading role. Nowadays, technology has redefined teaching in different areas and has played a crucial role innovating and transforming educational processes. This research describes the design of a web application as a learning strategy of surgical instruments usage inside the traumatology area. The development architecture was created by using only open source components, making this version freeware under any type of open source software license. Likewise, the state of the art of current surgical instruments available for teaching and training of medical personnel, specifically inside the traumatology area, is presented. This paper presents some final conclusions that should be taken into account when developing this type of applications.
\end{abstract}

Recibido: 16 de Octubre de 2013

Key words: Web application, serious games, surgical instruments, surgical training. 


\section{Introducción}

Hasta hace unas décadas, el entrenamiento quirúrgico solo era basado en los conocimientos y destrezas de sus tutores, de los cuales se aprende primero viendo, después ayudando y posteriormente haciendo, y donde la adquisición de habilidades y destrezas manuales están directamente relacionadas a una exposición constante y repetida de ellas (Graue, 2006). Con la convergencia de las nuevas tecnologías, unido a los avances en informática y las telecomunicaciones con la medicina, en general, $y$ la cirugía, en particular, se ha dado como resultado un proceso acelerado de informatización de todas las áreas de la medicina y, particularmente, en las especialidades quirúrgicas (Rodríguez, 2010).

Los juegos serios en computador son una herramienta que a través de la historia han ido ganando más y más importancia en distintos campos de la educación; no son solo vistos como juegos en sí, sino también como simuladores de un mundo real y tangible. En la actualidad existen diferentes áreas donde estos juegos son utilizados como complemento al modelo de enseñanza tradicional, como son: las ciencias de la salud, las diferentes ramas de las ingenierías, la formación militar y empresarial entre otras. Hoy día se abre la posibilidad de utilizarlos como una poderosa herramienta de aprendizaje para los estudiantes de diferentes programas. Estos juegos incluyen diversos beneficios pedagógicos y ayudan a desarrollar habilidades cognitivas (Leal et al., 2010).

En el presente trabajo se describe el diseño de una aplicación web como estrategia para el aprendizaje de instrumental quirúrgico en el área de traumatología. La arquitectura de desarrollo solo incluyó componentes de código abierto, por lo que esta versión es totalmente software libre y se puede liberar con cualquier tipo de licencia de código abierto.

En el siguiente apartado se describen brevemente algunas herramientas que se han venido incorporando en la enseñanza y entrenamiento de procedimientos quirúrgicos, enfocados en el área de la traumatología, tales como: robots, equipos de simulación, las tecnologías de información y comunicación, TIC, junto con los videojuegos y juegos serios. Luego se muestra la metodología seguida en el diseño y desarrollo de la aplicación web para el aprendizaje de material e instrumental quirúrgico, se presentan las evidencias del trabajo realizado y se termina con las conclusiones y algunas recomendaciones sobre la aplicación.

\section{Estado del Arte}

En los siguientes apartados se aborda el estado del arte de las herramientas y tecnología utilizada en el área de la cirugía, especialmente aquellas orientadas a la formación de personal médico en el área de la cirugía traumatológica.

\subsection{Los Robots}

La cirugía robótica comienza en 1985 con el Puma 560, un robot usado para realizar biopsias neuroquirúrgicas con mayor precisión. Tres años después, fue usado para llevar a cabo una prostatectomía transuretral. Después, la empresa Integrated Surgical Supplies Ltd., construyó dos modelos con características similares llamados: Probot, un robot diseñado específicamente para la prostatectomía transuretral, y Robodoc, un sistema robótico para realizar el vaciado del fémur con mayor precisión en operaciones de sustitución de cadera.

En 1998 se lanza al mercado el robot Zeus, con el cual se introduce el concepto de telerobótica o telepresencia en la cirugía robótica. Este robot se compone de una consola de control para el cirujano, con un sistema de vídeo tridimensional que proyecta imágenes desde una determinada distancia y una mesa operatoria con tres brazos robóticos con cuatro rangos de movimiento. Los brazos derecho e izquierdo simulan los brazos del cirujano, mientras que el tercer brazo es un 
endoscopio robótico Aesop controlado por voz. El principal inconveniente de Zeus es el gran tamaño de los brazos robóticos, el cual limita el espacio de las salas quirúrgicas y provocan colisiones, junto con el uso de gafas especiales (Valero et al., 2011).

Con los adelantos de la ciencia, surge en 1999 el robot quirúrgico Da Vinci, como el sistema de cirugía robótica más completo y desarrollado hasta hoy. Consta de tres componentes: el carro de visualización, la consola de cirujano y el carro móvil. El sistema fue diseñado para mejorar la laparoscopia tradicional y proporcionar la comodidad del cirujano, proporcionando una visualización de imágenes en tercera dimensión, con mayor destreza y mayor precisión, además de permitir realizar procedimientos mínimamente invasivos abarcando disecciones o reconstrucciones complejas. El sistema Da Vinci tiene como inconvenientes su tamaño, la gran cantidad de conexiones que se encuentran en la sala de operaciones que pueden causar accidentes, y el desmonte de los brazos robóticos en algunas intervenciones quirúrgicas (Valero et al., 2011).

En la actualidad compañías como Mimic Technologies, Inc. crean robots diseñados para la formación virtual y cirugía robótica con el objetivo de impartir clases a cirujanos que estén aprendiendo a operar con el sistema quirúrgico $\mathrm{Da}$ Vinci (Najarian et al., 2011). Según Valero et al. (2011), la cirugía robótica se convertirá en un nuevo medio para adquirir las habilidades necesarias para operar, gracias a la simulación de todas las intervenciones que pueden realizarse con un robot. Los cirujanos pueden usar robots quirúrgicos para practicar operaciones con simuladores tridimensionales de realidad virtual y modelos de las partes blandas que recrean la textura de los tejidos humanos a través de sistemas de respuesta de fuerza.

\subsection{La simulación}

El entrenamiento basado en simulación, es uno de los medios de enseñanza más utilizados hoy día en el área quirúrgica. La simulación tiene el potencial de complementar los recursos que son cada vez más escasos para la práctica de las competencias profesionales. Subespecialidades como oftalmología, ortopedia y la intervención vascular han aceptado el desafío de la simulación (Rosen, 2008). En la simulación quirúrgica generalmente se combinan artefactos de software y de hardware, que sumergen a la persona en un mundo virtual, dando sensaciones casi reales.

Bao \&Wang (2008), desarrollaron un framework de código abierto para realidad virtual basado en otros frameworks de simulación quirúrgica. El sistema está orientado a dos tipos de usuarios, uno es el cirujano en prácticas y otro es el experto o desarrollador, quien es responsable de configurar alguna aplicación a través del framework. Hutchins et al. (2005) presentan un sistema de entrenamiento para la cirugía del hueso temporal, con una herramienta de mano guiadora, que permite al instructor extender la mano para agarrar el extremo de la herramienta del estudiante, creando una conexión táctil de doble sentido entre ellos a través de la red. El instructor puede utilizar esta herramienta para guiar al alumno a determinados lugares dentro de la escena o para mostrar direcciones de accidente cerebrovascular. El inconveniente de esta propuesta, es que tanto instructor como estudiante deben estar en la misma habitación, lo que impide la enseñanza a distancia. Zhaoliang et al. (2011), construyen un sistema en tercera dimensión de rastreo y posicionamiento de instrumentos quirúrgicos utilizando un computador y tres cámaras para capturar las imágenes de los instrumentos quirúrgicos en tiempo real, basándose en el método de visión estereoscópica. Después de una serie de procesamiento de información que incluye, la calibración de las cámaras y la reconstrucción de las coordenadas en tercera dimensión de los instrumentos simulados, se obtienen 6 grados de libertad en los instrumentos simulados con un error absoluto de menos de $1 \mathrm{~mm}$. 
Sourina et al. (2007), implementan un programa simulador llamado "Virtual Bone-Setter", para ayudar a entender las relaciones tridimensionales complejas entre huesos e implantes. En este sistema, el cirujano localiza el hueso fracturado en el espacio en tercera dimensión observando la pantalla, luego, dependiendo de la fractura, el cirujano puede dar comandos para manipular los instrumentos y poner los implantes. El programa permite extender fácilmente a cualquier conjunto de huesos e implantes y herramientas, puesto que todos los objetos se almacenan en formato abierto de datos RWX, como también exportar las escenas a Virtual Reality Modeling Language (VRML), formato de datos para la visualización a través de internet y ser utilizados en entornos virtuales de enseñanza.

Los desarrollos en la simulación de cirugía traumatológica han sido orientados a la familiarización del aprendiz con el instrumental quirúrgico, así como a la creación de modelos anatómicos de gran calidad, permitiendo un mayor acercamiento a la realidad. Este es el caso del simulador "insightArthroVR", creado por Fernández et al. (2008), para el aprendizaje y entrenamiento de la cirugía artroscópica; en él se incluye instrumental simulado, en forma y textura, y se incorporan todas las funcionalidades y grados de libertad reales para la formación; el aprendiz va desarrollando habilidades, de forma progresiva, y se permite registrar los resultados y compararlos con un patrón de referencia, para obtener indicadores de destreza.

Acosta \& Muñoz (2011), proponen un sistema de simulación virtual basado en tecnología háptica, para el entrenamiento en los remplazos articulares de rodilla. Por medio de esta tecnología se logra la simulación de cortes e interacción con eliminación de partículas y representación de fuerzas en función de las superficies que entran en contacto. El sistema permite hacer cortes sobre modelos óseos en imágenes en tercera dimensión, similares a los que se realizan en el reemplazo de rodilla; también ofrece la oportunidad de elegir el tamaño de los componentes protésicos y su correcta alineación articular. La curva de aprendizaje viene dada por sesiones de entrenamiento con los residentes y el desarrollo de evaluaciones periódicas.

Vázquez \& Guillamet (2009) señalan que, la simulación quirúrgica viene dada por la reproducción completa de un quirófano, en el que utilizándose modelos anatómicos humanos o bien animales de experimentación, se entrenan procedimientos completos o situaciones quirúrgicas no previstas. Quirate \& Muñoz (2013), argumentan que, uno de los objetivos de la simulación es servir como herramienta didáctica del componente sicomotor en el cirujano, al trasladar una parte importante de la curva de aprendizaje del quirófano al laboratorio, reduciendo así la tasa de errores y complicaciones en beneficio de la seguridad del paciente. En el mismo sentido Silveira et al. (2012), concluyen en su investigación que, la simulación a través de los avances computacionales revolucionará la enseñanza de la cirugía y de otras especialidades de la medicina.

La simulación en cirugía se centra en adquirir las competencias profesionales necesarias sin someter a los pacientes a ningún riesgo. El entrenamiento basado en la simulación debe enmarcarse en una teoría que permita escalar los medios de simulación adecuados, como habilidades psicomotrices elementales, el aprendizaje de procedimientos quirúrgicos completos, el pensamiento crítico y la toma de decisiones (Vázquez, 2009; Roque et al. 2011), en su investigación concluyen que la simulación es un método de enseñanza y de aprendizaje muy útil en el desarrollo de la cirugía mínimamente invasiva, donde su empleo permite la adquisición de habilidades, convirtiéndose también en un elemento evaluativo en el proceso docente, que brinda ventajas para educandos, profesores y para los pacientes. Igualmente, García et al. (2011), señalan que las observaciones realizadas por expertos en el campo de la cirugía han permitido 
mejorar los prototipos de simuladores, para dar respuesta a las necesidades y expectativas de los estudiantes de cirugía y cirujanos que requieran mejorar su desempeño por medio del entrenamiento.

\subsection{Sistemas de planificación quirúrgica}

Existen diferentes proyectos software enfocados a la planificación de cirugías; así, González et al. (2013), presentan un sistema orientado a la planificación quirúrgica ortopédica en el área pediátrica, que permite tomar distintas mediciones especializadas, divididas en las regiones anatómicas, como son: cadera, rodilla y pie, entre otras. Con este sistema, el cirujano puede manipular las imágenes radiológicas hasta obtener la claridad requerida y el nivel de detalle deseado, en cuanto al estado de las articulaciones y los huesos. Además podrá realizar mediciones especializadas que le permitan obtener una visión más clara del procedimiento a realizar y aplicar filtros a las imágenes (paso bajo, paso alto e invertir los colores). La aplicación también puede utilizarse para uso docente de residentes en período de formación y para la formación de especialistas.

Otro sistema para este propósito es "Traumaplan”, diseñado por (Ramírez \& Coto, 2011), enfocado en fracturas de los miembros inferiores, el cual recibe como entrada una imagen radiográfica de la fractura de un paciente y pasa por otras etapas como medición y calibración, entre otras, hasta llegar a la segmentación y ensamble de la fractura. Las imágenes se obtienen usando una cámara digital a partir de una placa sobre un negatoscopio. El sistema usa una librería o repositorio donde se almacenan diversos implantes disponibles para la planificación.

\subsection{Las TIC en el entrenamiento quirúrgico}

La tecnología actual, permite utilizar distintos tipos de medios audiovisuales que dirigidos desde un computador resultan potentes instrumentos de enseñanza teórico-prácticos. El software hace posible el desarrollo de un entorno multimedia destinado a la docencia con las aplicaciones denominadas hipertexto e hipermedia. La multimedia fue uno de los primeros sistemas interactivos de enseñanza que incluían videodiscos con imágenes de diversas afecciones del aparato locomotor (Lacleriga, Cara \& Forriol, 1991). En la actualidad aún se siguen utilizando estos medios e incorporando otros como: videoconferencias, teleconferencias, aulas virtuales y cursos a distancia (Veloz et al., 2012). En el estudio realizado por Leal et al. (2010), sobre las tecnologías de información y comunicación en posgrados de salud, se demuestra la capacidad que tienen las TIC para producir innovación y colaboración, donde es necesario también para la docencia conocer los usos reales y cotidianos y contraponerlos con los proyectos institucionales y pedagógicos. En este sentido, desde hace varios años algunas Facultades de Medicina han incorporado módulos virtuales que dan apoyo a la educación presencial (Pi et al., 2004). Según Cejas \& Picorel (2009), integrar las TIC en el aula (virtual) requiere de un cambio de metodologías de aprendizaje, lo cual representa para los profesores un desafío y también una oportunidad de revisar los contenidos curriculares para darles mayor versatilidad, agilidad y practicidad.

Nuevos conceptos como los objetos de aprendizaje y el "e-learning" han surgido en los últimos años, los cuales dan soporte a la educación a distancia, agrupando herramientas o aplicaciones como parte fundamental en los procesos de enseñanza aprendizaje (De La Torre \& Domínguez, 2012). El centro de recursos de Internet creado por Citak et al. (2009), para la cirugía traumatológica, utiliza un Content Management System (CMS), el sistema Ilamado "Trauma Surgery Schoolbook", fue adoptado por primera vez en octubre de 2005 por la Escuela de Medicina de Hannover (Alemania) y desde entonces ha sido utilizado y evaluado por estudiantes de la institución. Con este sistema los estudiantes tienen acceso a los materiales desde el 
principio de semestre, y los instructores pueden editar y actualizar el material en cualquier momento. El inconveniente de esta propuesta es que solo muestra estadísticas de uso y no se puede evidenciar una curva de aprendizaje real.

Córdova (2012), diseña una herramienta de elearning, llamada "eHECT", para la cirugía traumatológica con los siguientes componentes: objeto de aprendizaje, repositorio de objetos educativos, herramienta de autoría, herramienta colaborativa y evaluativa, aplicaciones administrativas y un simulador de realidad virtual. La herramienta que se propone se sustenta en emplear herramientas que se integren para fortalecer la formación y capacitación integral del cirujano traumatólogo en el desarrollo de sus competencias. Este proyecto es totalmente software libre ya que todos sus componentes son de código abierto.

El internet ha posibilitado un gran número de alternativas de capacitación en el campo quirúrgico, brindando desde interacción con profesionales o participación en foros, hasta entrenamiento y educación en línea. Algunos sitios web que apoyan la formación en el área de la traumatología son: www.orthochina.org, es un sitio web con el concepto de Wiki, sin ánimo de lucro, dedicado a la cirugía ortopédica, donde los contenidos son creados en colaboración con usuarios y cirujanos especializados; los datos se clasifican en categorías y subespecialidades, y funciona como una plataforma de intercambio académico que proporciona libros electrónicos, videos y material didáctico en diferentes formatos (Ma et al., 2008). www.aofoundation.org, dispone de varios módulos de e-learning y cursos online en el área de la traumatología, además cuenta con transmisiones interactivas en vivo de procedimientos clínicos entregados en directo desde el quirófano (AO Fundation, 2013); www.ortho.hyperguides.com, creado en 2009 por Vindico Medical Education, es un sitio web que cuenta con una completa colección de tutoriales en línea, conferencias y ensayos; los tutoriales proporcionan información de características clínicas, radiológicas, diagnóstico de factores de riesgo, evaluación, tratamiento y resultados de los pacientes, además está acreditado por el Consejo de Acreditación para Educación Médica Continua (ACCME).

En el estudio realizado por Coughlan \& Brinkman (2011), señalan que son pocas las evaluaciones realizadas específicamente en términos de usabilidad y aprendizaje al incorporar las TIC en el entrenamiento quirúrgico. Este estudio concluye que las tecnologías basadas en internet pueden aprovechar plenamente el uso de contenidos multimedia, sin embargo, se deben tener en cuenta ciertas consideraciones de diseño para proporcionar auténticos ambientes de aprendizaje. Pillai \& Dennick (2012) argumentan que, existen carencias pedagógicas y subutilización de la tecnología Web 2.0 en la educación quirúrgica, asimismo, Ruiz et al. (2012), en su investigación plantean que la libre interacción de los estudiantes con materiales multimedia (no siempre de calidad) puede proporcionar aprendizajes incompletos, con visiones superficiales de la temática.

Otras propuestas formuladas y adoptadas por algunos investigadores para entrenar personal en el área de la cirugía, consisten en la creación de laboratorios utilizando elementos muy básicos. El estudio de (Rodríguez, 2003), se basa en un laboratorio docente para entrenamiento artroscópico, donde el proceso práctico ofrece un modelo progresivo de adiestramiento que comienza por el conocimiento del instrumental, observación directa en maquetas didácticas de la anatomía y ejecución de la técnica artroscópica bajo condiciones prácticamente similares a la realidad. Con la utilización de maquetas didácticas de diferentes articulaciones y un artroscopio completo, que incluye: lente, cámara, fuente de luz y monitor, el aprendiz realiza, bajo la supervisión del instructor, procedimientos quirúrgicos bajo condiciones que asemejan la realidad. De esta forma se entrena en la realización de diferentes 
vías de acceso a las articulaciones y se utiliza instrumental similar al que se emplea en los quirófanos reales. Con este modelo de enseñanza se ha apreciado un mejor desempeño del estudiante en el salón de operaciones, cuidando el instrumental costoso, conocer las normas técnicas de su utilización y sobre todo, la rápida obtención de habilidades que ha permitido la introducción de diversas técnicas y aplicación de tratamientos en hombro, codo y otras regiones anatómicas.

\subsection{Los videojuegos y los juegos serios}

El juego es una actividad fundamental para el desarrollo humano y el objetivo principal de quien lo practica es divertirse. Diversas investigaciones han demostrado las ventajas que tienen las personas que practican los videojuegos, como son: desarrollo en la coordinación de ojo y mano, mayor agudeza visual, rapidez de reacción, capacidad de atención a múltiples estímulos, facilidad para relacionarse con otros, alta motivación al logro, mayor tolerancia a la frustración, capacidad para tomar riesgos, resolver problemas y tomar decisiones (Marcano, 2008). En el área médica se han utilizado diferentes modalidades con el fin de complementar los procesos de enseñanza y aprendizaje en cirugía. En algunos establecimientos médicos y educativos de Estados Unidos cuentan con diversos equipos en las que el médico tiene que ir ejecutando paso a paso una cirugía virtual, "como un juego de Nintendo", hasta otros que apuntan a ejercitar su habilidad y precisión manual; en este proceso los residentes deben cumplir con cierto número de horas para luego ser evaluados (Cunzolo, 2012).

En los últimos años, ha surgido una nueva corriente llamada "juegos serios", los cuales son usados para entrenar y capacitar al personal en diferentes áreas, incluyendo la técnica quirúrgica. Rodríguez, (2012) desarrolló un juego serio de código abierto para la educación y entrenamiento en el procedimiento de artroscopia de rodilla. El sistema proporciona animaciones y simulaciones dinámicas para cuerpos blandos, partículas y fluidos. El juego presenta un entorno tridimensional que simula un quirófano con todos los elementos necesarios para la práctica de la artroscopia, el aprendiz, guiado por el "personaje" de un cirujano experto puede recorrer el quirófano y desarrollar el procedimiento. En este proyecto, el procedimiento quirúrgico siempre está sujeto a la memorización de los pasos, lo que hace que el juego se vuelve predecible.

De Paolis (2012), diseñó y desarrolló un prototipo de juego serio, para el entrenamiento en la sutura de la cirugía laparoscópica, para evaluar el nivel de habilidad desarrollado por los estudiantes. El juego utiliza dispositivos hápticos para simular la manipulación de instrumentos quirúrgicos, usa el motor de gráficos es Ogre 3D, Nvidia PhysX para el modelado físico de los objetos y la biblioteca de Application Programing Interface (HAPI) de Java para las interacciones hápticas. El sistema permite la simulación tanto de objetos rígidos como blandos, incluyendo telas y fluidos. La interfaz háptica se utiliza para simular la fuerza de respuesta del usuario en la manipulación de los instrumentos. El juego se utiliza para evaluar las habilidades en la realización de una sutura laparoscópica por medio de un hilo y dos abrazaderas controlados por dos interfaces hápticas. Los objetivos del sistema son: adquirir una buena coordinación ojo-mano, mejorar la capacidad de manipulación de instrumentos quirúrgicos y aprender las técnicas para realizar el nudo de sutura. Una desventaja del proyecto es que no almacena los resultados de las sesiones de entrenamiento, por lo tanto no se pueden evaluar los progresos en el entrenamiento.

\section{Diseño y desarrollo de una aplicación web}

En los apartados anteriores se mencionaron algunas ventajas que tienen hoy día los juegos serios en los procesos de enseñanza aprendizaje; esto se logra siempre y cuando el diseño y desarrollo del juego obedezca a estrategias pedagógicas y didácticas, que posibiliten en los 
estudiantes obtener un conjunto de conocimientos y competencias en un área específica. La eficacia de los juegos serios como objeto de aprendizaje reutilizable, reside en el proceso de creación del juego. Tanto el diseñador como el pedagogo desempeñan un papel muy importante en esta etapa. El proceso de creación de los juegos serios se divide en tres etapas: análisis contextual, desarrollo metodológico y evaluación. La primera y la última necesitan trabajo en equipo, mientras que la metodología se elabora individualmente según las premisas acordadas durante el análisis del contexto formativo (Sánchez, 2013).

El objetivo de la aplicación aquí reportada es permitir a los estudiantes de las escuelas de enfermería, así como a los profesionales sanitarios, repasar y tratar de memorizar el nombre del material quirúrgico necesario para cada intervención en el área de traumatología y lograr montar una mesa quirúrgica correcta, con base en los conocimientos teóricos adquiridos previamente.

\subsection{Modelado de la aplicación}

\subsubsection{Diseño conceptual}

El diseño y el desarrollo de la aplicación se basan en la teoría de los juegos serios; con esto se busca que la persona que desee practicar sus conocimientos teóricos, lo haga por medio de un juego, de una manera divertida, para alcanzar el objetivo de montar una mesa quirúrgica correcta.

En el diseño conceptual de la aplicación se utilizó el Lenguaje Unificado de Modelado, LUM o UML, del inglés Unified Modeling Language, el cual es capaz de abstraer cualquier tipo de sistema, informático o no, mediante diagramas que contienen toda la información relevante. En este lenguaje, un "Actor" puede ser una "Persona" o una "Cosa" del mundo real o abstracto que interacciona con el sistema que se está desarrollando (Cientec, 2013).
En la aplicación se pueden identificar dos actores: El primero es el "Estudiante", que asume el rol de "Jugador"; este actor puede ser un estudiante, un profesor, un enfermero o cualquier persona que desee practicar algún conocimiento en el juego. El segundo actor es el "Administrador", quien tiene los privilegios para hacer configuraciones en el juego, tales como: actualizar o asignar instrumental para los procedimientos quirúrgicos, consultar estadísticas del juego, asignar la duración en tiempo para la preparación de la mesa, y si lo desea, puede asumir también el rol de "Jugador".

\subsubsection{Modelo funcional}

En la figura 1 se presenta el diagrama funcional del juego, donde la implementación representa el procedimiento quirúrgico del cual se desea preparar la mesa de instrumental; luego, sigue con las fases del procedimiento elegido, que se clasifican en: preoperatoria, intraoperatoria y posoperatoria (Díaz, 2010), posteriormente se pasa a la selección del material y finaliza con la presentación de resultados.

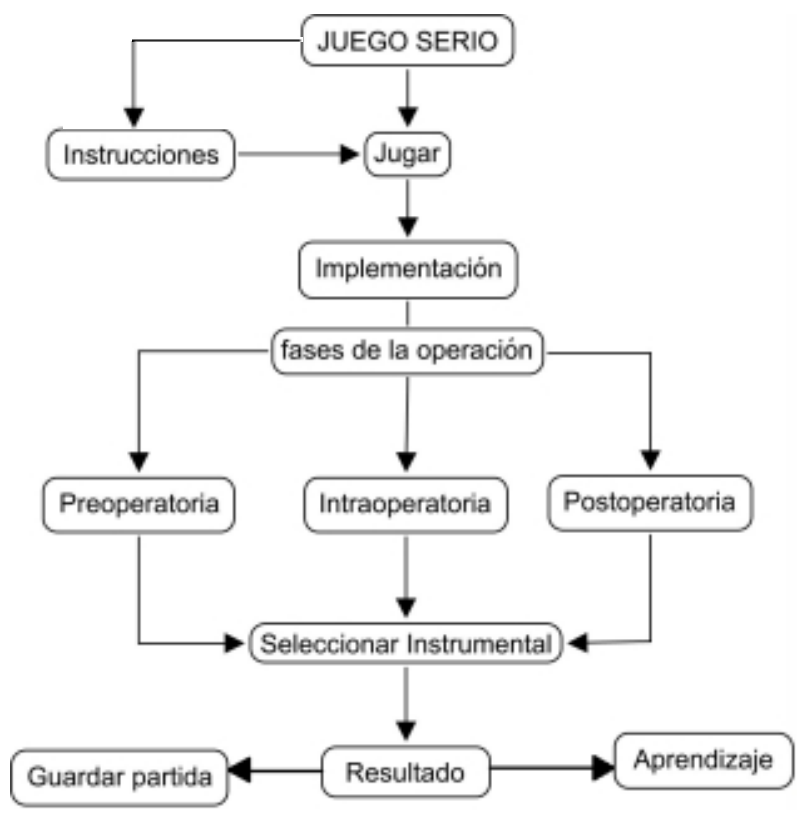

Figura 1. Diagrama funcional del Juego 


\subsubsection{Casos de uso}

En la figura 2 se muestran los casos de uso que intervienen en el sistema con sus dos "Actores" (estudiantey administrador).

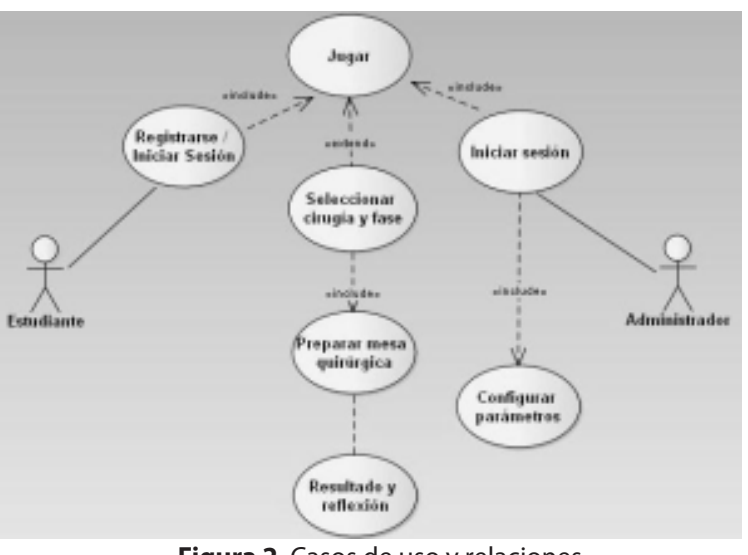

Figura 2. Casos de uso y relaciones

\subsection{Desarrollo}

\subsubsection{Arquitectura de desarrollo}

La aplicación tiene una arquitectura de desarrollo orientado a la web 2.0 (Ketterl, Mertens \& Vornberger, 2008), la cual es soportada por los navegadores, Mozilla Firefox, Internet Explorer, entre otros. El servidor web utilizado es Apache (proyecto Open Source) que provee arquitectura cliente/servidor, donde el cliente por medio de un navegador web se comunica con el servidor y éste da respuesta a sus peticiones utilizando el protocolo HiperText Transfer Protocol (HTTP) que está basado en el envío de mensajes (The Apache Software Foundation, 2012). El motor de base de datos utilizado es MySQL que tiene doble licencia: la licencia General Public License (GPL) que se puede adquirir como un producto de código abierto o la licencia comercial estándar de Oracle (2013). Los lenguajes de programación usados son: PHP, que es un lenguaje que permite crear páginas web dinámicas y que utiliza una sintaxis de programación orientada a objetos similar a la de Java (Sintes, 2013), Javascript, utilizado para crear algunos efectos especiales en las páginas y por último, tenemos a HTML5, que brinda la facilidad de integrar el contenido de vídeo directamente desde páginas web sin necesidad de utilizar un plug-in adicional (Yan et al., 2012).

\subsubsection{Pruebas}

La mecánica del juego se inicia a partir del registro o ingreso del usuario como se muestra en la figura 3 , luego se ingresa al panel principal donde se puede elegir una de las siguientes opciones: jugar, ver las instrucciones o consultar el ranking, ver figura 4.

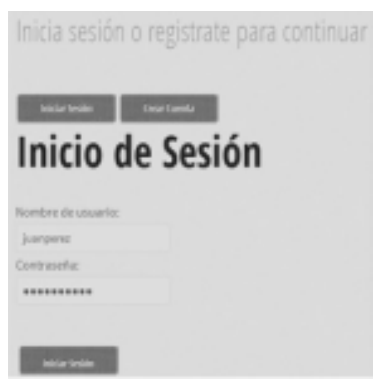

Figura 3. Inicio de sesión

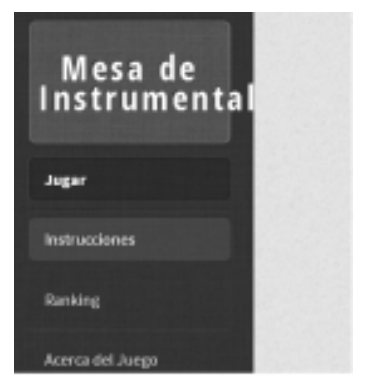

Figura 4. Opciones del juego
La aplicación tiene las opciones para realizar diferentes configuraciones del juego como: agregar o modificar instrumental para cada intervención, asignar tiempos aproximados para cada preparación de las mesas, entre otras. En la opción de jugar se debe elegir el procedimiento quirúrgico y la fase en la cual se va a preparar la mesa (preoperatoria, intraoperatoria y postoperatoria), como se observa en la figura 5. En la figura 6 se muestra un ejemplo del funcionamiento del juego en la "Cirugía férula en dedo" en la etapa intraoperatoria, la cual tiene un tiempo máximo de preparación de la mesa de 4 minutos y 30 segundos. El instrumental cargado en la parte izquierda de la figura 6 se debe arrastrar

\section{Seleccione el procedimiento quirúrgico}

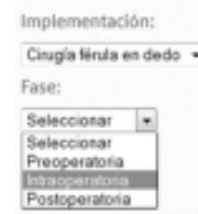

\section{Jugur}

Figura 5. Selección del procedimiento quirúrgico 
hasta la mesa de instrumental (rectángulo central) teniendo en cuenta que no todo el instrumental es correcto. El parámetro para cargar el instrumental en el juego se configura previamente, en este caso el total del instrumental correcto es del $50 \%$. Al terminar el juego, ya sea por tiempo o por elección del jugador se presenta el resultado obtenido. La partida es guardada para las estadísticas y posteriormente estos datos son el mecanismo que permitirá medir la curva de aprendizaje de cada participante.

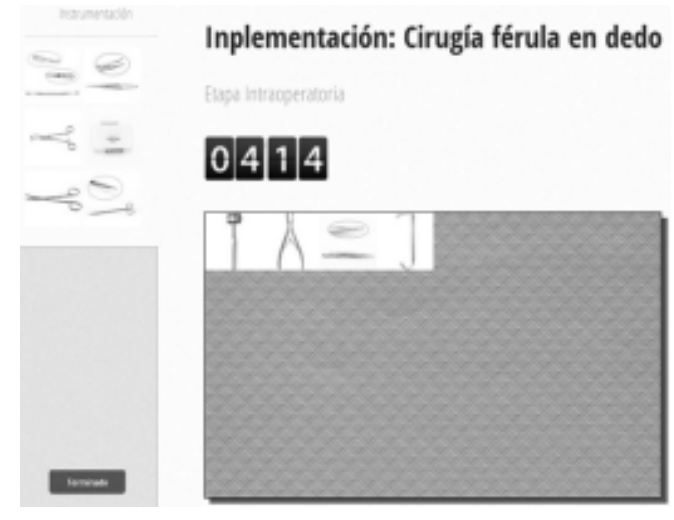

Figura 6. Colocación del material en la mesa

\section{Conclusiones}

Es de resaltar el valor que tienen las opiniones verbales de un cirujano experto, como el modelo principal de enseñanza de las habilidades quirúrgicas, por consiguiente, se hace necesario que se disponga de él en los programas de entrenamiento quirúrgico, sin embargo, la asignación de expertos y de espacios apropiados para la enseñanza son un reto cada vez mayor por costos y tiempo (Sánchez, 2007). Por lo anterior, han surgido diferentes propuestas que solucionan en parte este problema, evidenciándose un mayor desarrollo en el campo de la simulación y en menor proporción los denominados "Juegos Serios", no obstante, existe el interés de algunas organizaciones, universidades y grupos de investigación para aprovechar este medio de enseñanza-aprendizaje.

En los últimos años el uso de los juegos serios en los procesos de enseñanza-aprendizaje es creciente, ya que favorece la creatividad, la imaginación, facilita el mejor entendimiento de conceptos y proporciona entornos donde se pueden cometer errores y el aprender "haciendo" (Romero \& Turpo, 2012), asimismo, el juego desarrollado, brinda al usuario la facilidad de disponer de la herramienta en cualquier lugar por medio de un computador y un acceso a internet sin incurrir en costos adicionales.

En los proyectos de juegos serios deben existir claras metodologías para su diseño y desarrollo; los diseños deben adaptarse a la enseñanza y a la formación para luego ser utilizados efectivamente; sin embargo, siempre se requiere de un proceso de validación y de evaluación en un entorno real para probar su efectividad (Sánchez, 2013). Medina \& Zambrano (2010) argumentan que, los entornos como la web 2.0 fomentan el aprendizaje, motivan el trabajo en equipo e imparten conocimiento con un mejor uso del tiempo. Así también, el uso de herramientas web 2.0 pueden mejorar el estado de la enseñanza, el aprendizaje y la investigación en los sistemas de elearning (Rohani \&Yazdani, 2012).

La aplicación web aquí presentada es un prototipo, diseñado y desarrollado con el objetivo principal del aprendizaje de material o instrumental quirúrgico necesario para un determinado procedimiento. Vale la pena aclarar que en el Juego los materiales quirúrgicos no se ubican en la mesa, siguiendo algunas normas existentes para ello, de acuerdo con el procedimiento quirúrgico a realizar (Sappia, 2012); por lo anterior, como trabajo y desarrollo futuro quedará adaptar la aplicación para seguir algunas de estas normas. Otro aspecto a mejorar es el diseño en las interfaces, de modo que se facilite una mayor atención por parte de los usuarios. Una ventaja de la aplicación es que su arquitectura de desarrollo solo incluye componentes de código abierto, por lo tanto esta versión es totalmente software libre y se puede liberar con cualquier tipo de licencia de código abierto. 


\section{Referencias}

Acosta, J., \& Muñoz, E. (2011). Simulación virtual con tecnología háptica: entrenamiento para la cirugía de rodilla. Repertorio de medicina y cirugía, 20(4), 245-250.

AO Fundation. (2013). AO elearning: AOTrauma Principles Program eLearning. Recuperado el 16 de septiembre de 2013, de https://www.ao foundation.org/Structure/education/onlinecme/Pages/eLearning.aspx.

Bao, C., \& Wang, B. (2008). A open source based general framework for virtual surgery simulation. IEEE, 1(1), 575-579.

Cejas, C., \& Picorel, J. (2009). El empleo de las nuevas tecnologías en la enseñanza y el aprendizaje (TICS). Revista argentina de radiología, 73(2), 205-211.

Cientec. (2013). UML: Un lenguaje modelo. Recuperado el 8 de octubre de 2013, de http://ww w.cientec.com/analisis/ana-uml.html.

Citak, M., Calafi, A., Kendoff, D., Kupka, T., Haasper, C., Behrends, M., ... Hüfner, T. (2009). An internet based learning tool in orthopaedic surgery: Preliminary experiences and results. Technol Health Care, 17(2), 141-148. DOI: 10.3233/THC2009-0539.

Córdova, M. (2012). Diseño de una herramienta de e-learning para el área de cirugía traumatológica. Barcelona, España: Universidad Oberta de Catalunya, Tesis de Maestría.

Coughlan, C., \& Brinkman, W. (2011). Design considerations for delivering e-learning to surgical trainees. International journal of ehealthand medical communications, 2, 14-23.

Cunzolo, F. (2012). Operaciones virtuales y simulacros, las nuevas herramientas de los cirujanos. Recuperado el 22 de mayo de 2013, de http://www.semanaprofesional.com/?nota=34132.

De La Torre, L., \& Domínguez, J. (2012). Las TIC en el proceso de enseñanza aprendizaje a través de los objetos de aprendizaje. Revista Cubana de Informática Médica,4(1), 1-15.

De Paolis, L. (2012). Serious Game for Laparoscopic Suturing Training. Sixth International Conference on Complex, Intelligent, and Software Intensive Systems, 481-485, Lecce, Italy.

Díaz, A. (2010). Verificación del cumplimiento de la lista de chequeo para minimización de eventos quirúrgicos adversos transoperatorios: Prácticas para una cirugía más segura. Ciencia y Salud, 2(1), 43-60.

Fernández, J., Cuervo, P., Illana, A., Pérez, P., Rodríguez, B., \& Bayona, S. (2008). Entrenador Simulador Avanzado para el aprendizaje y entrenamiento de la Cirugía Artroscópica. Trauma Fundación MAPFRE, 19(1), 50-58.

García, J., Arias, M., \& Valencia, E. (2011). Diseño de prototipo de simulador para entrenamiento en cirugía laparoscópica. Ingeniería Biomédica, 5(9), 13-19.

González, Y., Cartelle, M., \& López, Y. (2013). Sistema para la planificación quirúrgica ortopédica en el área de pediatría, IX Congreso Internacional de Informática en Salud, La Habana, Cuba.

Graue, E. (2006). La enseñanza de la cirugía. Recuperado el 12 de agosto de 2013, de http://www.facmed.unam.mx/eventos/seam2k1/ 2006/oct_02_ponencia.html.

Hutchins, M., Stevenson, D., Gunn, C., Krumpholz, A., Adriaansen, T., Pyman, B., \& O'Leary, S. (2006). Communication in a networked haptic virtual environment for temporal bone surgery training. Virtual Reality, 9 (2), 97-107. DOI: 10.1007/s10055005-0015-1 
Ketterl, M., Mertens, R., \& Vornberger, O. (2008). Web Lectures and Web 2.0. Tenth IEEE International Symposium on Multimedia, 720725, California, EE. UU.

Lacleriga, A., Cara, J., \& Forriol, F. (1991). Nuevos aportes de la informática aplicada a la docencia en Cirugía Ortopédica y Traumatología. Revista Española de Cirugía Osteoarticular, 26(156), 303305.

Leal, M., Viladrich, C., Murat, M., \& Caminal, J. (2010). Tecnologías de información y comunicación en instituciones de posgrados de salud: evidencias y estereotipos. Revista de Universidad y Sociedad del Conocimiento, 7(1), 112.

Ma, Z., Zhang, H., Yu, T., Ren, T., Du, G., \& Wang, Y. (2008). Case-based Orthopaedic Wiki Project in China. Clinical Orthopaedics and Related Research, 466(10), 2428-2437.

Marcano, B. (2008). Juegos serios y el entrenamiento en la sociedad digital. Revista Electrónica Teoría de la Educación, 9(3), 93-107.

Medina, V., \& Zambrano, W. (2010). Elearning model for the higher education based on web 2.0. International Conference on Education and Management Technology, 51-54, Cairo, Egypt.

Molina, J., Silveira, E., Heredia, D., Fernández, D., Bécquer, L., Gómez, T., ... Castro, M. (2012). Los simuladores y los modelos experimentales en el desarrollo de habilidades quirúrgicas en el proceso de enseñanza-aprendizaje de las Ciencias de la Salud. Revista electrónica de veterinaria, 13(6), 1-23.

Najarian, S., Fallahnezhad, M., \& Afshari, E. (2011). Advances in medical robotic systems with specific applications in surgeryare view. Journal of Medical Engineering \&Technology, 35(1), 19-33.
Oracle. (2013). MySQL: The world's most popular open source database. Recuperado el 9 de octubre de 2013, de http://dev.mysql.com/doc/refman /5.1/en

Pi, F., Sueiras, A., Gil, G., \& Jaurrieta, E. (2004). La asignatura virtual. "Fundamentos de Cirugía en Internet". Educación Médica, 7(1), 24-29.

Pillai, J., \& Dennick, R. (2012). Contemporary pedagogy with insurgical education websites: a review of the literature. International Journal of Medical Education, 3, 21-36.

Quirarte, C., \& Muñoz, J. (2013). La revolución pedagógica en la cirugía, Parte III. Metodología de la enseñanza de destrezas psicomotoras: los simuladores. Revista Mexicana de Cirugía Endoscópica, 14(1), 37-51.

Ramírez, E., \& Coto, E. (2011). Traumaplan: para la planificación preoperatoria en traumatología. Revista Venezolana de Información, Tecnología y Conocimiento, 8(2), 61-78.

Rohani, M., \& Yazdani, K. (2012). Web 2.0 Embedded E-Learning: A Case Study. International Conference on Innovation, Management and Technology Research, 603-608, Malacca, Malaysia.

Rodríguez, C. (2003). Laboratorio docente para entrenamiento artroscópico. Educación Médica Superior, 17(3).

Rodríguez, F. (2012). Serious game de código abierto para la educación y entrenamiento en el procedimiento de artroscopia de rodilla. M.S. Tesis, Universidad Oberta de Catalunya, Barcelona, España.

Rodríguez, Z. (2010). Cualidades necesarias en el cirujano. Revista Cubana de Cirugía, 49(3). Recurerado el 8 de agosto de 2013, de http://bvs.sld.cu/revistas/cir/ 
Roque, R., Fernández, A., Martínez, M., Torres, R., López, A., \& Barrios, I. (2011). Entrenamiento basado en la simulación para la formación en cirugía mínimamente invasiva. Educación Médica Superior, 25(3), 320-325.

Romero, M., \&Turpo, O. (2012). Serious Games para el desarrollo de las competencias del siglo XXI. Revista de educación a distancia, (34), 1-22.

Rosen, K. (2008). The history of medical simulation. Journal of Critical Care, 23, 157-166.

Ruiz, A., Ángel, E., \& Guevara, O. (2009). La simulación clínica y el aprendizaje virtual: tecnologías complementarias para la educación médica. Revista Facultad de Medicina de la Universidad Nacional de Colombia, 57(1), 67-79.

Sánchez, M. (2007). Formación y adiestramiento en traumatología y cirugía ortopédica. Revista Española de Cirugía Osteoarticular, 42(232), 139180.

Sánchez, M. (2013). Buenas Prácticas en la Creación de Serious Games: Objetos de Aprendizaje Reutilizables, Recuperado el 8 de octubre de 2013, de http://spdece07.ehu.es/ actas/Sanchez.pdf.

Sappia, D. (2012). Guía de Estudios de Cirugía General: Mesa del instrumental. Recuperado el 21 de octubre de 2013, de: http://www.vet.unicen. edu.ar/html/Areas/Cirugia\%20general/Documen tos/8-Mesainstrlnstru mentac.pdf

Silveira, E., Heredia, D., Fernández, D., Bécquer, L., Gómez, T., González, Y., et al. (2012). Los simuladores y los modelos experimentales en el desarrollo de habilidades quirúrgicas en el proceso de enseñanza-aprendizaje de las Ciencias de la Salud. Revista electrónica de veterinaria, 13(6), 1-23.
Sintes, B. (2013). Páginas web con PHP. Extraído el 9 de octubre de 2013, de http://www.mclibre.org/ consultar/php

Sourina, O., Sourin, A., \& Sen, H. (2007). Orthopedic surgery training simulation. Journal of Mechanics in Medicine and Biology, 7(1), 37-53.

The Apache Software Foundation. (2012). Apache: HTTP server project. Recuperado el 8 de octubre de 2013, de http://httpd.apache.org

Valero, R., Koa, Y., Chauhan, S., Schatloff, O., Sivaraman, A., Coelho, R., et al. (2011). Cirugía robótica: Historia e impacto en la enseñanza. Actas Uralógicas Españolas, 35(9), 540-545.

Vázquez, G. (2009). Simulación, utilidad en la formación médica y quirúrgica. Cirugía Española, 86(1), 1-2.

Vázquez, G. \& Guillamet, A. (2009). El entrenamiento basado en la simulación como innovación imprescindible en la formación médica. Fundación educación médica, 12(3), 149155.

Veloz, M., Almanza, E., Uribe, J., Díaz, L., Quintana, V., \& Alanís, P. (2012). Uso de tecnologías en información y comunicación por médicos residentes de ginecología y obstetricia. Revista de investigación en educación médica, 1(4), 183-189.

Vindico Medical Education. (2009). Orthopedics hyperguide. Recuperado el 18 de septiembre de 2013, de http://www.ortho.hyperguides.com.

Yan, X., Yang, L., Lan, S., \& Tong, X. (2012). Application of HTML5 Multimedia. Computer Science and Information Processing, 871-874.

Zhaoliang, D., Zhiyong, Y., Xiangyun, L., Weixin, S., \& Jianhui, Z. (2011). 3D Tracking and Positioning of Surgical Instruments in Virtual Surgery Simulation. Journal of Multimedia, 6(6), 502-509. 